\title{
A NOTE ON THE RESTRICTION THEOREM AND GEOMETRY OF HYPERSURFACES
}

\author{
FABIO NICOLA
}

\begin{abstract}
A necessary condition is established for the optimal $\left(L^{p}, L^{2}\right)$ restriction theorem to hold on a hypersurface $S$, in terms of its Gaussian curvature. For some classes of flat hypersurface we give sharp thresholds for the range of admissible exponents $p$, depending on the specific geometry.
\end{abstract}

\section{Introduction and discussion of the results}

Consider a smooth hypersurface $S \subset \mathrm{R}^{n}$, and a compactly supported continuous function $0 \leq \psi \in C_{c}(S)$. Let $d \mu=\psi d \sigma$, where $d \sigma$ is the measure induced by the Lebesgue one. The celebrated restriction theorem by Stein-Tomas for the Fourier transform (we use the standard notation $\hat{f}(\xi)=\int e^{-i x \xi} f(x) d x$ ) states that, provided the Gaussian curvature $K$ of $S$ does not vanish on the support of $\psi$, one has the estimate

$$
\left\|\left.\widehat{f}\right|_{S}\right\|_{L^{2}(S, d \mu)} \leq A\|f\|_{L^{p}\left(\mathrm{R}^{n}\right)}, \quad \forall f \in \mathscr{S}\left(\mathrm{R}^{n}\right),
$$

for every

$$
1 \leq p \leq \frac{2 n+2}{n+3}
$$

and for some constant $A>0$ depending on $S, \psi$ and $p$ (see [14], [11] and also [13] for a survey of the restriction problem). We observe that in the SteinTomas restriction theorem a certain degree of smoothness is required. To our knowledge, it is not known whether the conclusion still holds under the assumption of a lower bound on the Gaussian curvature (defined geometrically) alone. On this point, we refer to the recent paper by Iosevich and Sawyer [8], where an example is constructed of a convex surface in $\mathrm{R}^{n}$ with curvature bounded from below and, nevertheless, the decay of the Fourier transform is sub-optimal.

Here we are interested in the following related question: what constraints does the validity of (1.1) impose on the geometry of $S$ and on the density $\psi$ ?

Received November 27, 2006; in revised form January 23, 2007. 
The answer of course depends on the choice of the Lebesgue exponent $p$ in (1.1). A recent result by Iosevich and $\mathrm{Lu}$ [7] states that if (1.1) holds with the optimal $p=(2 n+2) /(n+3)$ then $S$ must have non-zero curvature where $\psi \neq 0$. The following theorem, being quantitative in nature, extends that result.

THeOREM 1.1. There exists a universal constant $c>0$ such that, if (1.1) holds with $p=(2 n+2) /(n+3)$ then

$$
\psi(x) \leq c A^{2}|K(x)|^{\frac{1}{(n+1)}}, \quad x \in S .
$$

It is worth mentioning the uniformity of this estimate with respect to the objects involved.

Also, Theorem 1.1 has some consequences for estimates which contain a power of the curvature as a mitigating factor, like

$$
\left\|\left.\widehat{f}\right|_{S}|K|^{s} \phi\right\|_{L^{2}(S, d \sigma)} \leq A\|f\|_{L^{p}\left(\mathrm{R}^{n}\right)}, \quad \forall f \in \mathscr{S}\left(\mathrm{R}^{n}\right), \quad p=\frac{2 n+2}{n+3},
$$

with $\phi \in C_{c}^{\infty}(S)$ and $s \geq 0$. Such an estimate with $s=1 /(n+1)$ was proved to be true by Sjölin [10] (see also [15]) for $n=2$ and every $S$ convex, and recently by Carbery and Ziesler [1] in some cases with $n \geq 2$ (see also Cowling et al. [2] for related decay estimates for the Fourier transform). As explained in [1], the choice of the exponent $s=1 /(n+1)$ is mandated by affine invariance considerations. On the other hand, as a consequence of Theorem 1.1 applied with $\psi(x)=|K(x)|^{s}|\phi(x)|, \phi \in C_{c}^{\infty}(S)$, one deduces the following fact:

Suppose there is a point $\bar{x} \in S$, with $\phi(\bar{x}) \neq 0, K(\bar{x})=0$, that is the limit of points at which the curvature is non-zero. Then (1.3) cannot hold unless $s \geq 1 /(n+1)$.

Notice that the above mentioned result by Sjölin shows that Theorem 1.1 is sharp.

We now fix the attention on the case in which there are some vanishing curvatures. It follows from [7] that if at some point $\bar{x} \in S$ with $\psi(\bar{x}) \neq 0$ there are $k$ vanishing principal curvatures then (1.1) cannot hold unless $p \leq$ $2(n-k / 3+1) /(n-k / 3+3)$. However, if the other $n-1-k$ curvatures are not zero at $\bar{x}$ one can only say, in general, that (1.1) holds with $p=$ $2(n-k+1) /(n-k+3)$, as shown by Greenleaf [4]. It is observed in [4] that this latter result is sharp when $S$ contains an open subset which is the product of a $(n-1-k)$-dimensional surface with nonzero Gaussian curvature cross a piece of $k$-dimensional plane. In the following theorem we prove that such a value of $p$ indeed represents a threshold in more general situations. 
Let $v(x), x \in S$, be the number of the principal curvatures which vanish at $x$, namely the dimension of the kernel of the second fundamental form at $x$.

Theorem 1.2. Let $\bar{x} \in S$, with $\psi(\bar{x}) \neq 0$ and let $\underline{v}:=\liminf _{x \rightarrow \bar{x}} v(x)$. Then the estimate in (1.1) cannot hold unless

$$
p \leq \frac{2(n-\underline{v}+1)}{n-\underline{v}+3} .
$$

When $\underline{v}=0$ the conclusion of Theorem 1.2 is a consequence of Knapp's optimality result (see Lemma 3 of Strichartz [12]). When $\underline{v}>0$ the proof is a combination of a scaling argument with a reduction of $S$ to a kind of normal form (see Proposition 2.2 below). This auxiliary result follows from some facts of Riemannian geometry of hypersurfaces in Euclidean space and can be of some interested in its own right, though similar Morse-type reductions are quite common in the context of Fourier integral operators and in partial differential equations, see e.g. Hörmander's book [5].

We also deduce a consequence for decay estimates for the Fourier transform of the measure $d \mu=\psi d \sigma$, i.e. estimates of the form

$$
|\widehat{d \mu}(\xi)| \leq C(1+|\xi|)^{-r}, \quad r>0 .
$$

It is well known that (1.5) holds with $r=(n-1-k) / 2$ if $S$ has $n-1-k$ non-vanishing curvatures on the support of $\psi$. In all cases, (1.5) implies the restriction estimate (1.1) for $p \leq 2(r+1) /(r+2)$ (see [4]), whereas the converse was proved by Iosevich [6] for smooth convex hypersurfaces of finite type, in the sense that the order of contact with every tangent line is finite (see also [7], [9], [11] for related results).

As a consequence of Theorem 1.2 we deduce the following fact:

Let $\bar{x} \in S$, with $\psi(\bar{x}) \neq 0$ and let $\underline{v}:=\liminf _{x \rightarrow \bar{x}} v(x)$. Then (1.5) cannot hold unless $r \leq(n-1-\underline{v}) / 2$.

ACKNOWLedgments. I wish to thank Professors Antonio J. Di Scala, Fulvio Ricci and Luigi Rodino for helpful discussions on the subject of this paper. I am also very grateful to Professor Anthony Carbery for providing his paper [1].

\section{Proof of the results}

Proof of Theorem 1.1. Assume (1.1) with $p=(2 n+2) /(n+3)$ and then observe that, by an application of the Fatou lemma, the estimate in (1.1) holds true for all $f \in L^{1} \cap L^{p}$ as well. Fix now two functions $f_{1}$ and $f_{2}$ 
in $L^{1}\left(\mathrm{R}^{n-1}\right) \cap L^{p}\left(\mathrm{R}^{n-1}\right)$ and $L^{1}(\mathrm{R}) \cap L^{p}(\mathrm{R})$ respectively, with $\widehat{f_{1}}>0$ and $\widehat{f}_{2}>0$, and set $f(x)=f_{1}\left(x^{\prime}\right) f_{2}\left(x_{n}\right), x=\left(x^{\prime}, x_{n}\right)$.

It suffices to prove (1.2) for those $x \in S$ such that $K(x) \neq 0$, since we already know from Theorem 2 of [7] that $\psi=0$ where $K=0$. Moreover it is easy to see that, if (1.1) holds, then it also holds, with the same constant $A$, for the surface $\Lambda(S)$ (with density $\psi \circ \Lambda^{-1}$ ) where $\Lambda: \mathrm{R}^{n} \rightarrow \mathrm{R}^{n}$ is any translation or rotation. Hence, we can take any point at which $K \neq 0$ and, possibly after a translation and rotation, we can assume such a point as the origin of the coordinates, with $S$ locally defined by the equation $x_{n}=\phi\left(x^{\prime}\right)$, $x^{\prime}$ in an open neighborhood $U \subset \mathrm{R}^{n-1}$ of 0 , with $\phi(0)=0, \nabla \phi(0)=0$, and $\phi^{\prime \prime}(0)$ in diagonal form. By (1.1) we hence obtain

$$
\left\|\tilde{\psi}\left(x^{\prime}\right) \int e^{-i\left(x^{\prime} \xi^{\prime}+\phi\left(x^{\prime}\right) \xi_{n}\right)} g(\xi) d \xi\right\|_{L^{2}\left(\mathrm{R}_{x^{\prime}}^{n-1}\right)} \leq A\|g\|_{L^{p}\left(\mathrm{R}^{n}\right)}, \quad p=\frac{2 n+2}{n+3},
$$

for every $g \in L^{1}\left(\mathrm{R}^{n}\right) \cap L^{p}\left(\mathrm{R}^{n}\right)$, where $\tilde{\psi}=\chi \psi^{1 / 2}$, for any cut-off function $\chi \in C_{c}^{\infty}(U), 0 \leq \chi \leq 1$. We take $\chi$ satisfying $\chi(0)=1$, so that $\tilde{\psi}(0)=$ $\psi(0)^{1 / 2}$.

Let $\kappa_{1}, \ldots, \kappa_{n-1}$ be the eigenvalues of $\phi^{\prime \prime}(0)$ (recall, $\kappa_{1} \kappa_{2} \cdots \kappa_{n-1}=$ $K(0) \neq 0)$. We apply the estimate (2.1) with

$$
g(x)=f_{\delta}(x):=f\left(\delta B^{-1 / 2} x^{\prime}, \delta^{2} x_{n}\right),
$$

where $\delta>0$ and $B=\operatorname{diag}\left[\left|\kappa_{1}\right|, \ldots,\left|\kappa_{n-1}\right|\right]$ (therefore $B^{-1 / 2}=\operatorname{diag}\left[\left|\kappa_{1}\right|^{-1 / 2}\right.$, $\left.\left.\ldots,\left|\kappa_{n-1}\right|^{-1 / 2}\right]\right)$. Then we obtain

$$
\begin{aligned}
\left\|\tilde{\psi}\left(x^{\prime}\right) \int e^{-i\left(\delta^{-1} B^{1 / 2} x^{\prime} \xi^{\prime}+\delta^{-2} \phi\left(x^{\prime}\right) \xi_{n}\right)} f(\xi) d \xi\right\|_{L^{2}\left(\mathrm{R}_{x^{\prime}}^{n-1}\right)} \\
\leq A \delta^{\frac{n+1}{p^{\prime}}}|K(0)|^{-\frac{1}{2 p^{\prime}}\|f\|_{L^{p}\left(\mathrm{R}^{n}\right)},}
\end{aligned}
$$

and therefore, after the change of variables $x^{\prime} \rightarrow \delta B^{-1 / 2} x^{\prime}$ (recall, $f=$ $\left.f_{1} \otimes f_{2}\right)$,

$$
\begin{aligned}
\delta^{\frac{n-1}{2}}|K(0)|^{-\frac{1}{4}}\left\|\tilde{\psi}\left(\delta B^{-1 / 2} x^{\prime}\right) \widehat{f_{1}}\left(x^{\prime}\right) \widehat{f_{2}}\left(\delta^{-2} \phi\left(\delta B^{-1 / 2} x^{\prime}\right)\right)\right\|_{L^{2}\left(\mathrm{R}_{x^{\prime}}^{n-1}\right)} \\
\leq A \delta^{\frac{n+1}{p^{\prime}}}|K(0)|^{-\frac{1}{2 p^{\prime}}}\|f\|_{L^{p}\left(\mathrm{R}^{n}\right)}
\end{aligned}
$$

Since $p^{\prime}=2(n+1) /(n-1)$, the exponents of $\delta$ in the two sides of (2.2) are equal. Now we observe that, as $\delta \rightarrow 0^{+}, \tilde{\psi}\left(\delta B^{-1 / 2} x^{\prime}\right) \rightarrow \psi(0)^{1 / 2}$ and

$$
\delta^{-2} \phi\left(\delta B^{-1 / 2} x^{\prime}\right) \rightarrow \sum_{j=1}^{n-1} \epsilon_{j} x_{j}^{2} / 2, \quad \epsilon_{j}=\operatorname{sign} \kappa_{j} .
$$


Hence, by the Fatou lemma ( $\hat{f}_{2}$ is continuous) we have

$$
\psi(0)^{1 / 2}|K(0)|^{-\frac{1}{4}+\frac{1}{2 p^{\prime}}} \leq c_{n} A,
$$

with

$$
c_{n}:=\sup _{\epsilon_{j}= \pm 1}\|f\|_{L^{p}\left(\mathrm{R}^{n}\right)}\left\|\widehat{f_{1}}\left(x^{\prime}\right) \widehat{f_{2}}\left(\sum_{j=1}^{n-1} \epsilon_{j} x_{j}^{2} / 2\right)\right\|_{L^{2}\left(\mathrm{R}_{x^{\prime}}^{n-1}\right)}^{-1} .
$$

In order to conclude the proof, it suffices to show that, for a convenient choice of $f_{1}, f_{2}$, the above sequence $c_{n}$ is bounded. To this end, choose $f_{1}\left(x^{\prime}\right)=e^{-\left|x^{\prime}\right|^{2} / 2}$, $f_{2}\left(x_{n}\right)=\left(1+x_{n}^{2}\right)^{-1}$. Then $\hat{f}_{1}\left(x^{\prime}\right)=(2 \pi)^{(n-1) / 2} e^{-\left|x^{\prime}\right|^{2} / 2}$ and $\hat{f}_{2}\left(x_{n}\right)=\pi e^{-\left|x_{n}\right|}$. Since $\left|\hat{f}_{2}\left(\sum_{j=1}^{n-1} \epsilon_{j} x_{j}^{2} / 2\right)\right| \geq \hat{f}_{2}\left(\left|x^{\prime}\right|^{2} / 2\right)$ it follows that

$$
c_{n}=\|f\|_{L^{p}\left(\mathrm{R}^{n}\right)}(2 \pi)^{-\frac{n-1}{2}} \pi^{-1}\left(\int e^{-2\left|x^{\prime}\right|^{2}} d x^{\prime}\right)^{-\frac{1}{2}} .
$$

Now, we have $\left\|f_{1}\right\|_{L^{p}\left(\mathrm{R}^{n-1}\right)}=(2 \pi / p)^{(n-1) /(2 p)}$ and $\left\|f_{2}\right\|_{L^{p}(\mathrm{R})} \leq \pi^{1 / p}$, hence

$$
c_{n} \leq \pi^{\frac{1}{p}-1}\left\{\left(\frac{2 \pi}{p}\right)^{\frac{1}{2 p}}(2 \pi)^{-\frac{1}{2}}\left(\frac{\pi}{2}\right)^{-\frac{1}{4}}\right\}^{n-1} .
$$

Finally, we observe that $p \rightarrow 2$ as $n \rightarrow+\infty$, so that $c_{n} \rightarrow 0$ and this concludes the proof.

We now prove some results needed for the proof of Theorem 1.2.

For $x \in S$, let $\alpha_{x}: T_{x} S \times T_{x} S \rightarrow \mathrm{R}$ be the second fundamental form of $S$ at $x$, so that $\nu(x)=\operatorname{dim}\left(\operatorname{Ker} \alpha_{x}\right)$.

Proposition 2.1. Let $\bar{x} \in S$ and $\underline{v}=\liminf _{x \rightarrow \bar{x}} v(x)$. There is an affine plane L of dimension $\underline{v}$ passing through $\bar{x}$, such that $S \cap L$ contains a relatively open ball $B$ of $L$ with center in $\bar{x}$ and

$$
\alpha_{x}(\mathbf{v}, \mathbf{w})=0, \quad \forall x \in B, \quad \mathbf{v} \in T_{x} L, \quad \mathbf{w} \in T_{x} S .
$$

Proof. First of all we observe that there is an open neighborhood $V \subset S$ of $\bar{x}$ such that $\underline{v}=\min _{x \in V} v(x)$.

Consider then the case in which $v(\bar{x})=\underline{v}$. Therefore $v(x)$ is constant for $x$ in an open neighborhood $U \subset S$ of $\bar{x}$. It is a classical result (see e.g. Theorem 5.3 of [3]) that the distribution $U \ni x \mapsto \operatorname{Ker} \alpha_{x}$ is (smooth and) integrable, and its leaves are totally geodesics in $S$ and in $\mathrm{R}^{n}$. Hence, the result follows by taking $L=\left\{x \in \mathrm{R}^{n}: x-\bar{x} \in \operatorname{Ker} \alpha_{\bar{x}}\right\}$.

Assume now $v(\bar{x})>\underline{v}$. Then there is a sequence of points $S \ni x_{j} \rightarrow \bar{x}$, $j \geq 1$, with $v\left(x_{j}\right)=\underline{v}$. By the first part of the present proof applied to $x_{j}$ 
there are affine planes $L_{j}$ and balls $B_{j} \subset L_{j}$ with center in $x_{j}$ satisfying the properties in the statement at $x_{j}$. Moreover a subsequence of $L_{j}$ tends to a plane $L$ of dimension $\underline{v}$ and containing $\bar{x}$. To conclude the proof, it is therefore sufficient to proof that the $B_{j}$ have radii bounded from below by a positive constant, for (2.3) then follows by continuity.

To this end we observe that every $B_{j}$ is the union of geodesics that are rays from $x_{j}$. On the other hand, it is a consequence of the Cauchy theorem for ordinary differential systems that geodesics starting from points sufficiently near $\bar{x}$, regardless of their starting directions, have lengths bounded form below by a positive constant.

Proposition 2.2. Let $\bar{x} \in S$ and $\underline{v}:=\liminf _{x \rightarrow \bar{x}} v(x)$. There is an orthonormal system of coordinates $x=\left(x^{\prime}, x^{\prime \prime}, x_{n}\right), x^{\prime}=\left(x_{1}, \ldots, x_{n-1-\underline{v}}\right)$, $x^{\prime \prime}=\left(x_{n-\underline{v}}, \ldots, x_{n-1}\right)$ with the origin at $\bar{x}$ such that, in a neighborhood of $\bar{x}$, $S$ is the graph of a function $x_{n}=\phi\left(x^{\prime}, x^{\prime \prime}\right)$ of the type

$$
\phi\left(x^{\prime}, x^{\prime \prime}\right)=\left\langle M\left(x^{\prime}, x^{\prime \prime}\right) x^{\prime}, x^{\prime}\right\rangle,
$$

where $M$ is a square matrix of size $n-1-\underline{v}$ with smooth entries.

Notice that, in general, $v(\bar{x}) \geq \underline{v}$, but the most interesting case in Proposition 2.2 is of course when $v(\bar{x})>\underline{v}$. Also, observe that the transformation of $\mathrm{R}^{n}$ which brings $S$ in the desired form is an orthogonal one, and not merely smooth.

Proof of Proposition 2.2. It is a direct consequence of Proposition 2.1. Indeed, after a translation and a rotation $S$ coincides with the graph of a function $x_{n}=\phi\left(x_{1}, \ldots, x_{n-1}\right)$, with $\phi(0)=0, \nabla \phi(0)=0$. Now, after a further rotation, one can take the plane $L$ in the statement of Proposition 2.1 as the coordinate plane of equation $x_{1}=\cdots=x_{n-1-\underline{v}}=x_{n}=0$. Then (2.3) gives

$$
\frac{\partial^{2} \phi}{\partial x_{j} \partial x_{k}}\left(0, x^{\prime \prime}\right)=0, \quad \text { if } n-\underline{v} \leq j<n \text { or } n-\underline{v} \leq k<n,
$$

namely $\nabla \phi\left(0, x^{\prime \prime}\right)=0$ for $x^{\prime \prime}$ small. Hence also $\phi\left(0, x^{\prime \prime}\right)=0$ for $x^{\prime \prime}$ small, and an application of the Taylor formula at $x^{\prime}=0$ yields (2.4).

Proof of Theorem 1.2. We consider the coordinate system centered at $\bar{x}$ given by Proposition 2.2, so that $S$ coincides, in neighborhood of the origin, with the graph of a function $x_{n}=\phi\left(x^{\prime}, x^{\prime \prime}\right)$ of the type (2.4) for $\left(x^{\prime}, x^{\prime \prime}\right)$ in an open neighborhood $U$ of the origin.

Suppose therefore that the estimate (1.1) holds for some $p \geq 1$. Then

$$
\left\|\tilde{\psi}\left(x^{\prime}, x^{\prime \prime}\right) \int e^{-i\left(x^{\prime} \xi^{\prime}+x^{\prime \prime} \xi^{\prime \prime}+\phi\left(x^{\prime}, x^{\prime \prime}\right) \xi_{n}\right)} f(\xi) d \xi\right\|_{L^{2}\left(\mathbb{R}_{x^{\prime}, x^{\prime \prime}}^{n-1}\right)} \leq A\|f\|_{L^{p}\left(\mathrm{R}^{n}\right)},
$$


where $\tilde{\psi}=\chi \psi^{1 / 2}$, for any cut-off function $\chi \in C_{c}^{\infty}(U), 0 \leq \chi \leq 1$, $\chi(0) \neq 0$.

We now choose two Schwartz functions $f_{1}$ and $f_{2}$ in $\mathrm{R}^{n-1}$ and $\mathrm{R}$ respectively, with $\widehat{f_{1}}>0, \widehat{f_{2}}>0$. Upon setting $f(x)=f_{1}\left(x^{\prime}, x^{\prime \prime}\right) f_{2}\left(x_{n}\right)$, we test (2.6) on the function $f_{\delta}(x)=f\left(\delta x^{\prime}, \delta^{\epsilon} x^{\prime \prime}, \delta^{2} x_{n}\right), \epsilon>0$. By arguing as in the proof of Theorem 1.1 we obtain

$$
\begin{aligned}
& \delta^{\frac{n-1-v+\epsilon \nu}{2}}\left\|\tilde{\psi}\left(\delta x^{\prime}, \delta^{\epsilon} x^{\prime \prime}\right) \widehat{f}_{1}\left(x^{\prime}, x^{\prime \prime}\right) \widehat{f}_{2}\left(\delta^{-2} \phi\left(\delta x^{\prime}, \delta^{\epsilon} x^{\prime \prime}\right)\right)\right\|_{L^{2}\left(\mathrm{R}_{x^{\prime}, x^{\prime \prime}}^{n-1}\right)} \\
& \leq A \delta^{\frac{n+1-\underline{\nu}+\epsilon \underline{\underline{\nu}}}{p^{\prime}}}\|f\|_{L^{p}\left(\mathrm{R}^{n}\right)} .
\end{aligned}
$$

Now, as $\delta \rightarrow 0$ we see that $\tilde{\psi}\left(\delta x^{\prime}, \delta^{\epsilon} x^{\prime \prime}\right) \rightarrow \chi(0) \psi^{1 / 2}(0) \neq 0$, whereas

$$
\left.\delta^{-2} \phi\left(\delta x^{\prime}, \delta^{\epsilon} x^{\prime \prime}\right)\right) \rightarrow\left\langle M(0,0) x^{\prime}, x^{\prime}\right\rangle .
$$

By dominated convergence it follows that the $L^{2}$ norm in the left hand side of (2.7) tends to a number $c \neq 0$. Hence, we see that necessarily

$$
p \leq \frac{2(n-\underline{v}+\epsilon \underline{v}+1)}{n-\underline{v}+\epsilon \underline{v}+3} .
$$

Since $\epsilon$ is arbitrary, we obtain (1.4).

\section{REFERENCES}

1. Carbery, A., Ziesler, S., Restriction and decay for flat hypersurfaces, Publ. Mat. 46 (2002), 405-434.

2. Cowling, M., Disney, S., Mauceri, G., Müller, D., Damping Oscillatory Integrals, Invent. Math. 101 (1990) 237-260.

3. Dajczer, M., Submanifolds and isometric immersions, Publish or Perish, Inc., Houston, 1990.

4. Greenleaf, A., Principal Curvature and Harmonic Analysis, Indiana Univ. Math. J. 30 (1990), 519-237.

5. Hörmander, L., The analysis of Linear Partial Differential Operators. IV. Fourier Integral Operators, Grundlehren Math. Wiss. 275 (1985).

6. Iosevich, A., Fourier transform, $L^{2}$ restriction theorem and scaling, Boll. Unione Mat. Ital. 2 (1999), 383-387.

7. Iosevich, A., Lu, G., Sharpness results and Knapp's homogeneity argument, Canad. Math. Bull. 43 (2000), 63-68.

8. Iosevich, A., Sawyer, E., Three problems motivated by the average decay of the Fourier tranform, Harmonic analysis at Mount Holyoke, Contemp. Math. 320 (2003).

9. Sogge, C. D., Fourier Integrals in Classical Analysis, Cambridge Tracts in Math. 105 (1993).

10. Sjölin, P., Fourier multpliers and estimates of the Fourier transform of measures carried by smooth curves in $\mathrm{R}^{2}$, Studia Math. 51 (1974), 169-182.

11. Stein, E. M., Harmonic analysis: real-variable methods, orthogonality, and oscillatory integrals, Princeton Math. Ser. 43. Monographs in Harmonic Analysis, III, 1993.

12. Strichartz, R. S., Restrictions of Fourier transforms to quadratic surfaces and decay of solutions of wave equations, Duke Math. J. 44 (1977), 705-714. 
13. Tao, T., Recent progress on the Restriction conjecture, Park City proceedings, to appear, Available at ArXiv, math.CA/0311181.

14. Tomas, P., A restriction theorem for the Fourier transform, Bull. Amer. Math. Soc. 81 (1975), 477-478.

15. Zigmund, A., On Fourier coefficients and transforms of functions of two variables, Studia Math. 50 (1974), 189-202.

DIPARTIMENTO DI MATEMATICA

POLITECNICO DI TORINO

CORSO DUCA DEGLI ABRUZZI

24 - 10129 TORINO

ITALY

E-mail: fabio.nicola@polito.it 